\title{
Telemedicine/E-health and the Soul of Medical Practice
}

ISSN: 2689-2707

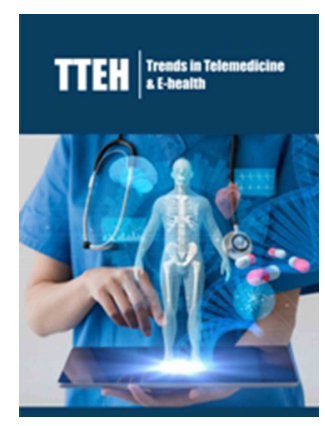

*Corresponding author: Eileen E Morrison, School of Health Administration, USA

Submission: 監 December 14, 2019

Published: 毕January 06, 2020

Volume 2 - Issue 2

How to cite this article: Eileen E M. Telemedicine/E-health and the Soul of Medical Practice. Trends Telemed E-Health 2(2). TTEH. 000533. 2020. DOI: 10.31031/TTEH.2020.02.000533

Copyright@ Eileen E Morrison, This article is distributed under the terms of the Creative Commons Attribution 4.0 International License, which permits unrestricted use and redistribution provided that the original author and source are credited.

\author{
Eileen E Morrison* \\ School of Health Administration, USA
}

\section{Opinion}

Telemedicine, E health and other technologies promise change that can profoundly affect patients and providers. Even a quick scan of topics featured in this journal demonstrates its impact on quality of life, public health concerns, and effectiveness healthcare. However, this powerful area of health care also creates serious issues for patients and providers. This writer is particularly interested in ethics issues that affect the soul of health care.

It is important to consider both patients and providers in this discussion. While it is true that patients come first, serving patients requires an effective, well regulated, and profitable system. From the business side, one hears-no money no care. How do differing views affect ethics as it relates to ethical practices for health technologies? First, consider the patient's experience. Patients learn about the miracles of health technology from medical shows, Internet sites and friends. When they face their own health crisis, there can be pain, disability, fear, and the threat of death. They want action now! In these situations, the best possible treatment is primary. Patients do not consider cost, insurance coverage or lack thereof. In their emotional situation, they depend on the ethics those who treat them and in the quality of the facility.

How does ethics apply to the patient and health technologies? A framework ethics basics can simplify this discussion and includes autonomy, beneficence, nonmaleficence, and justice. For example, patients, while the enjoying the convenience of telemedicine, may also have concerns about the safety of their medical information. They worry that systems can be hacked or that information is sold for marketing purposes. In some cases, fear makes patients reluctant to share vital information for successful treatment. In addition, patients are expected to comply with computer-based systems for making appointments, receiving health information, and other interactions with healthcare professionals. While these interactions are cost-effective and acceptable to many patients, they may be perceived as a violation of autonomy. Patients may prefer human interaction to computers.

Beneficence and nonmaleficence may be also be considered for health technology. Some patients readily accept telemedicine and $\mathrm{E}$ health innovations with a positive view of their benefit. Others view the use of multiple technologies as a lack of respect for the person and a sign of they are becoming "just another number". In these circumstances, the practitioner's ability to communicate on a factual and compassionate level is essential. With respect to nonmaleficence, patients may question the benefits of telemedicine and $\mathrm{E}$ health. This can occur if errors are made by the "computer" than end in loss of information, need for repeated tests, or other outcomes. Justice for the patient may be linked access and costs of telemedicine and $\mathrm{E}$ health initiatives. Because patients are often dealing with their emotions as well as they are diagnoses, they are not always thinking of fairness to others. For example, they are not concerned with the cost benefits of technology to the organization that serves them. They do not consider utilization of staff and the cost of updating information systems. Rather, their concerned about why some patients appear to have access to life-saving technology while they do not. When it is their child, mother, or other loved ones, they expect fairness as they conceive it to be. 
Providers also face issues related to health technology. Like any business, they depend on the creating profit and maintaining their reputation to attract customers. To maintain their "center of excellence" status, they may have "state of the art" services and the latest technologies. However, unlike other businesses, their finances come from direct pay, insurance reimbursement, and government. Because of the nature of the business, they must comply with regulation from multiple sources. Providing care also requires employees with a wide variety of expertise, which adds to the cost of doing business. Health technology assists with marketing and the efficiency of business. In addition, they are mandated to use technology to their regulation requirements and payment for services.

Does health technology affect the soul of health care in its business practices? Again, the framework of autonomy, beneficence, nonmaleficence, and justice provides basis for discussion. The benefits of technology for patient care also creates a duty toward autonomy. For example, healthcare businesses must design processes and procedures for accurate information and to safeguard the privacy of that information. This means investment in employees training, updating systems, and compliance with HIPPA rules. These efforts require a commitment of the organization's culture and finances. Beneficence and nonmaleficence are also part of business of health care. While technologies become familiar and comfortable for providers, the patient may have concerns. Beneficence requires making the time to explain, reassure, and practice respect for patients when using technology. Organizations should stress the need for many forms of beneficence in their mandatory orientation and training programs. In addition, nonmaleficence is included in the organization's policies and procedures for both patients and personnel. This includes verification of current licensure and continuing education. To encourage the application of this principle, the organization provides training on new or updated technology to reduce possible harm.

The principle of justice is essential for a successful healthcare organization. First, those who provide services to patients expect to be treated with fairness by their employers. The consideration for fair treatment is included in business practices, employee policies and procedures, and government mandates. In addition, healthcare organizations need to maintain an image of fairness within their communities and among their supporters. As in any business, actions and word of mouth are important to community status and business success. Therefore, healthcare organizations must be active in hearing and resolving patient concerns. They must also practice sincere concern and take appropriate action when needed. There may be a financial cost for practicing justice for employees and patients, but the cost benefit of maintaining a reputation for justice is worth the investment.

How do healthcare technologies affect the soul of health care? In many ways, they can improve quality of life and increase prevention outcomes. They also have the potential to reduce the human factor and create a hole in the soul of health care. This writer has great hope that the effective use of technology can benefit both the business of health care and those that it serves. The potential is exciting and worth exploring. However, it is important to remember that health care is about the human condition and requires a deep understanding of the importance of health in that condition. Therefore, the soul of health care should not be forgotten. Applying the basic principles of ethics is a first step in this significant process. 